\title{
Body Mass Index and Somatic Symptom Severity in Patients with Somatic Symptom Disorder: The Mediating Role of Working Memory
}

\author{
Hee Jin Kim', Hye Ri Kim', Jae-Chan Jin², Doug Hyun Han', Sun Mi Kim \\ ${ }^{1}$ Department of Psychiatry, Chung-Ang University College of Medicine, ${ }^{2}$ Department of Social Welfare, Graduate School of Soongsil University, \\ Seoul, Korea
}

\begin{abstract}
Objective: In somatic symptom disorder (SSD), cognitive dysfunction is related to perceptive distortion that excessively amplifies bodily sensations. The association between high body mass index (BMI) and cognitive dysfunction could be attributed to underlying systemic inflammation. We aimed to evaluate whether patients with SSD and high BMI exhibit increased somatic symptom severity and whether this is mediated by cognitive dysfunction.

Methods: This retrospective, cross-sectional study was conducted on 54 patients with SSD (20 males/34 females, mean age \pm standard deviation: $40.65 \pm 13.23$ years). Participants' BMI, laboratory data including complete blood count and lipid profile, results from the Korean Wechsler Adult Intelligence Scale-IV, and scores of the Korean-Symptom Checklist 95-Somatization (SCL95-SOM) were analyzed. We performed a path analysis to evaluate BMI as a predictor of somatic symptoms.

Results: In a path analysis, the SCL95-SOM score was directly influenced by working memory $(b=-0.326, p=0.032)$, which was significantly influenced by $\mathrm{BMI}(\mathrm{b}=-0.338, p=0.009)$, although there was no direct effect of $\mathrm{BMI}$ on the SCL95-SOM score. The path analytic model showed a close fit to the data with the following values: $\chi^{2}(\mathrm{df})=$ $0.918(1), p=0.338$, root mean square error of approximation $=0.000(<0.001)$, and comparative fit index $=1.00$. Conclusion: Patients with SSD and high BMI may exhibit increased somatic symptom severity, and this is mediated by working memory dysfunction. Weight management may help improve symptoms in patients with SSD and high BMI.
\end{abstract}

KEY WORDS: Somatoform disorder; Body mass index; Working memory; Cognitive dysfunction.

\section{INTRODUCTION}

Patients with somatic symptom disorder (SSD) exhibit multiple somatic symptoms that are distressing, but not necessarily related to identified medical conditions [1]. They have excessive thoughts, feelings, and behaviors related to somatic symptoms and visit multiple doctors for similar symptoms, resulting in considerable healthcare burdens [1-3]. Although the etiology of SSD remains unclear [4], one explanation is a faulty cognitive schema,

Received: June 8, 2020 / Revised: July 23, 2020

Accepted: July 30, 2020

Address for correspondence: Sun Mi Kim

Department of Psychiatry, Chung-Ang University College of Medicine, 84 Heukseok-ro, Dongjak-gu, Seoul 06974, Korea E-mail: sunmikim706@gmail.com

ORCID: https://orcid.org/0000-0003-4131-0542 leading to patients focusing their attention on bodily sensations, misinterpreting them, and getting alarmed by them [5]. Thus, patients amplify their somatic sensations and have low tolerance for physical discomfort [5]. Deficits in several cognitive domains, such as working memory and attention, have been reported in SSD [6-13].

Obesity increases the risk of chronic medical illnesses including hypertension, diabetes mellitus, dyslipidemia, cardiovascular diseases, autoimmune diseases, and cancers [14-19]. Moreover, obesity is related to the major depressive and bipolar disorders [20-24], possibly through chronic low-grade systemic inflammation that affects brain function and causes emotional and cognitive changes [25-27].

Although several studies have focused on the association between increased body mass index (BMI) and mood

(c) This is an Open-Access article distributed under the terms of the Creative Commons Attribution Non-Commercial License (http://creativecommons.org/licenses/by-nc/4.0) which permits unrestricted non-commercial use, distribution, and reproduction in any medium, provided the original work is properly cited. 
disorders, few have investigated the association between $\mathrm{BMI}$ and SSD. Considering the underlying mechanism that obesity causes mood disorders by dysfunctions in the top-down regulation of emotion through connections with several brain structures [28], it is also compelling to assume that dysregulation of top-down process over cognitive function would lead to another neuropsychiatric disorder such as SSD [7]. Working memory difficulties are often reported in patients with SSD $[6,10]$. Therefore, we aimed to investigate the association between $\mathrm{BMI}$ and somatic symptoms and possible mediators. We hypothesized that patients with SSD and high BMI exhibit increased somatic symptom severity, mediated by cognitive dysfunction, specifically working memory dysfunction.

\section{METHODS}

\section{Study Participants and Data Collection}

We retrospectively extracted data of participants who visited the Department of Psychiatry, Chung-Ang University Hospital in Seoul, Korea, between June 1, 2013 and April 30, 2019. We included (1) patients aged between 20 and 65 years and (2) those diagnosed with SSD based on the Structured Clinical Interview for DSM-5-Clinician Version [29]. We excluded the following patients: (1) those with past or current episodes of any other psychiatric diseases, including neurocognitive disorder and intellectual disability; (2) those with a past or current diagnosis and/or pharmacological treatment of chronic or severe medical illnesses including malignancy, hematologic disease, cardiovascular disease, stroke, and rheumatic disease; (3) those with a history of psychotropic medication use during the last month; and (4) those with a history of head trauma. The first data search included 64 patients with SSD. Ten patients were excluded because of incomplete data. Finally, 54 patients with SSD were included in the statistical analyses.

\section{Measures and Procedure}

\section{Biomedical factors}

Patients' weight and height were measured by nurses, and BMI was computed. Glucose, low-density lipoprotein cholesterol, triglyceride (TG), and total cholesterol levels and white blood cell and absolute neutrophil counts (ANCs) were measured using standardized procedures at the Depart- ment of Diagnostic Tests, Chung-Ang University Hospital.

\section{Neurocognitive factors}

We used the Korean Wechsler Adult Intelligence Scale-IV, which is designed to assess one's intellectual abilities within four domains [30,31]: (1) Verbal comprehension is the capability to listen to information provided orally and then remember, understand, and use the information; (2) perceptual reasoning is the mental capacity to think and reason using visual information; (3) working memory is short-term memory with limited capacity to hold information temporarily and is important for learning, reasoning, and guidance for decision-making and action; and (4) processing speed is the speed at which cognitive processes and responses occur.

\section{Somatic symptom severity}

The Korean-Symptom Checklist 95-Somatization (SCL95-SOM) was used to evaluate the severity of distress arising from somatic symptoms [32]. The 12 items of the SCL95-SOM reflect the distress caused by perceptions of bodily dysfunction, including strong autonomic mediation and muscle pain. The items are scored using a 4-point Likert scale (1 ["not at all"] to 4 ["almost always"]); we used the T-scores of the sum of the 12 item scores.

\section{Statistical Analyses}

Descriptive statistics for each characteristic were calculated and tested for normality using the Shapiro-Wilk test. Because BMI, TG, ANC, and SCL95-SOM score distributions were positively skewed, these variables were log-transformed. Preliminary analyses were performed to characterize the nature of the cross-sectional association among the factors of interest. We performed bivariate (Pearson) correlations among BMI, TG, ANC, total intelligence quotient $(\mathrm{IQ})$, verbal comprehension, perceptual reasoning, working memory, processing speed, and SCL95-SOM score. To evaluate BMI as a predictor of somatic symptoms, we performed a path analysis using IBM SPSS Amos version 24.0 (IBM Co., Armonk, NY, USA). Model fit was assessed by examining the chi-squared $\left(\chi^{2}\right)$ statistic ( $p>0.05$ was considered acceptable), root mean square error of approximation (RMSEA; $<0.08$ was acceptable), and comparative fit index ( $\mathrm{CFl}$; values close to 1 indicated a good fit). 


\section{Ethics Statement}

The research protocol was approved by the Chung-Ang University Hospital Institutional Review Board (no. 1908003-16275). Informed consent from the participants was waived because the study was conducted retrospectively.

\section{RESULTS}

\section{Characteristics of Study Participants}

Demographic factors, somatic symptom severity, neurocognitive factors, biomedical factors, past medical history, and behavioral factors of the participants are described in Table 1.

\section{Preliminary Analyses}

Bivariate correlations performed to examine the crosssectional associations among the factors of interest revealed that BMl correlated with TG $(r=0.387, p=0.004)$, ANC ( $r=0.393, p=0.003)$, total IQ $(r=-0.303, p=$ 0.026), verbal comprehension $(r=-0.350, p=0.009)$, and working memory $(r=-0.302, p=0.026)$. Additionally, the SCL95-SOM score correlated with working memory ( $\mathrm{r}=$ $-0.306, p=0.025)$. For other factors, there were no significant correlations.

\section{Path Analyses}

We constructed a path analytic model to evaluate BMI as a predictor of somatic symptom severity and vice versa. The model showed a close fit to the data with $\chi^{2}(\mathrm{df})=$ $0.918(1), p=0.338$, RMSEA $=0.000(<0.001)$, and CFI $=$ 1.00 (Fig. 1). The SCL95-SOM score was directly influ-
Table 1. Characteristics of study participants $(n=54)$

\begin{tabular}{|c|c|}
\hline Variable & Value \\
\hline \multicolumn{2}{|l|}{ Demographic factors } \\
\hline Age (yr) & $40.65 \pm 13.23$ \\
\hline Sex, female & 63.0 \\
\hline Education (yr) & $13.57 \pm 3.08$ \\
\hline Marriage, married & 44.4 \\
\hline Duration of SSD (mo) & $40.25 \pm 61.51$ \\
\hline \multicolumn{2}{|l|}{ Somatic symptom severity } \\
\hline SCL95-SOM score & $68.59 \pm 16.78$ \\
\hline \multicolumn{2}{|l|}{ Neurocognitive factors } \\
\hline Total IQ & $94.80 \pm 14.64$ \\
\hline Verbal comprehension & $97.67 \pm 15.53$ \\
\hline Perceptual reasoning & $95.89 \pm 16.21$ \\
\hline Working memory & $100.26 \pm 11.81$ \\
\hline Processing speed & $90.93 \pm 13.70$ \\
\hline \multicolumn{2}{|l|}{ Biomedical factors } \\
\hline Body mass index $\left(\mathrm{kg} / \mathrm{m}^{2}\right)$ & $22.25 \pm 3.72$ \\
\hline Random glucose (mg/dl) & $110.52 \pm 37.03$ \\
\hline LDL cholesterol (mg/dl) & $104.33 \pm 70.04$ \\
\hline Triglycerides (mg/dl) & $99.71 \pm 22.78$ \\
\hline Total cholesterol (mg/dl) & $171.68 \pm 33.53$ \\
\hline White blood cell count $\left(\times 10^{6} / \mathrm{L}\right)$ & $6,473.47 \pm 1,694.10$ \\
\hline Absolute neutrophil count $\left(\times 10^{6} / \mathrm{L}\right)$ & $3,645.92 \pm 1,360.02$ \\
\hline \multicolumn{2}{|l|}{ Past medical history } \\
\hline Diabetes & 11.1 \\
\hline Hypertension & 14.8 \\
\hline Dyslipidemia & 13.0 \\
\hline Thyroid disorder & 1.9 \\
\hline \multicolumn{2}{|l|}{ Behavioral factors } \\
\hline Smoking, current smoker & 13.0 \\
\hline Alcohol use & 31.5 \\
\hline
\end{tabular}

Values are presented as mean \pm standard deviation or \%. SSD, somatic symptom disorder; SCL95-SOM, symptom checklist 95somatization; IQ, intelligence quotient; LDL, low-density lipoprotein.

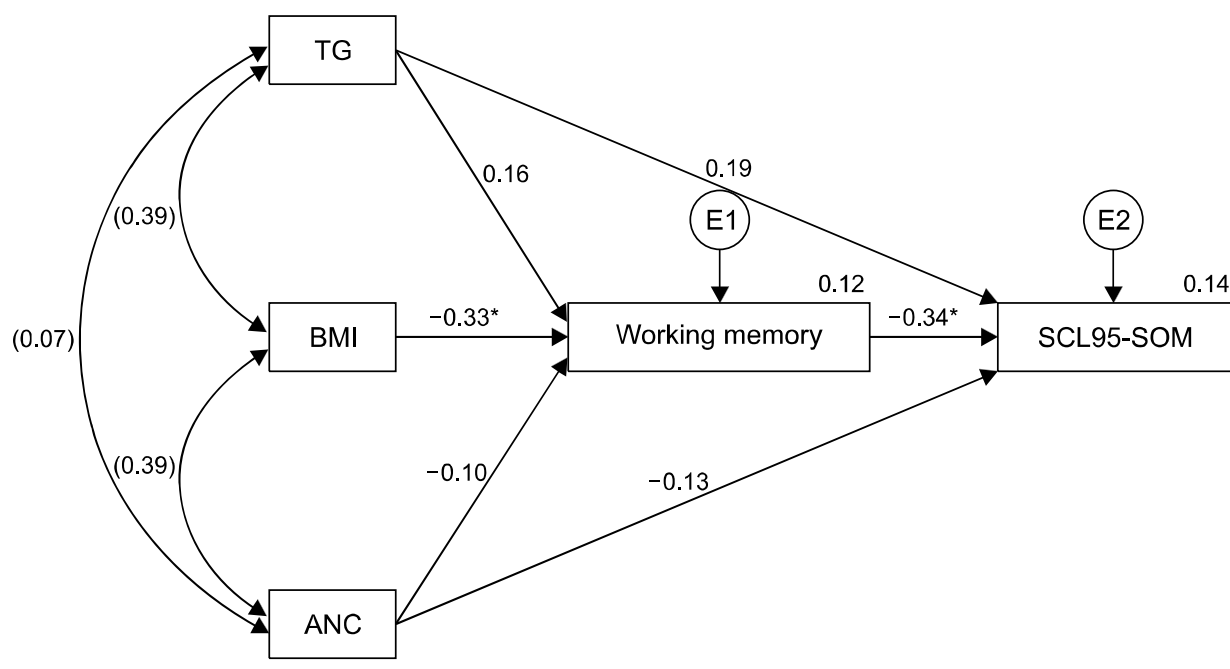

Fig. 1. Results of path analysis to test the integrated model. BMI, body mass index; TG, triglyceride; ANC, absolute neutrophil count; SCL95-SOM, symptom checklist 95-somatization; RMSEA, root mean square error of approximation; CFI, comparative fit index. Statistical significance, ${ }^{*} p<0.05$. $\chi^{2}(\mathrm{~d} f)=0.918(1), p=0.338$, RMSEA $=$ $0.000(<0.001), \mathrm{CFI}=1.00$. 
enced by working memory $(b=-0.326, p=0.032)$, which was significantly influenced by BMI $(b=-0.338, p=$ 0.009). We found no direct effect of BMl on the SCL95-SOM score.

\section{DISCUSSION}

The positive correlation between BMI and TG and between BMI and ANC observed in this study is consistent with previous findings that obesity increases the risk of metabolic dysfunction [15-18] and systemic inflammation [25-27]. Previous studies reported a significant association between inflammation and psychiatric symptoms [33-35]. Increased pain sensitivity was shown to be associated with increased concentrations of tumor necrosis factor- $\alpha(\mathrm{TNF}-\alpha)$ [36]. Obesity-related inflammation originating from the adipose tissue spreads to the brain and causes changes in neural circuits, neurotransmitter activity, neuroendocrine system, and neurogenesis $[37,38]$. Increased BMI is associated with detrimental changes in the white matter microstructure that supports working memory $[39,40]$.

However, we found no direct effect of BMI on somatic symptom severity. Instead, the positive association between BMI and somatic symptom severity was mediated by working memory, indicating that rather than directly affecting the autonomic nervous system, obesity causes systemic inflammation, affects brain regions associated with working memory, causes cognitive distortion [25-27], and worsens somatic symptoms. SSD patients showed reduced attention and increased functional connectivity between the sensorimotor and dorsal attention network [41]. Similarly, fibromyalgia patients showed reduced working memory, correlating with the frontal lobe and anterior cingulate gyrus volumes [42]. Working memory is the capability to keep information accessible over time and is closely related to attention as attention helps this process by preserving the information selectively [43]. Reduced working memory and attention suggests misunderstanding of external stimuli and failure to regulate functions aimed at interaction with external stimuli [41], which might lead to the development and worsening of somatic symptoms. Several studies reported co-occurrence of somatic symptoms and cognitive deficits [6-12], but the causality between them remained to be determined. However, the results of this study suggest that working memory may precede exacerbation of somatic symptoms, considering that the path analytic model implies a possible causal association. Previous findings that nociception could be modulated by cognitive factors support this association $[44,45]$.

Our study had some limitations. First, the sample size $(\mathrm{n}=$ 54) was relatively small. However, in path analysis, a sample size of 10 times the number of parameters is considered adequate [46]. In our study, the number of parameters was 5 , indicating a minimal sample size of 50 , which means that our sample size was appropriate. Second, we measured only ANC, not inflammation markers such as ESR, CRP, TNF- $\alpha$, and interleukins because they are not included in routine laboratory tests in clinical settings. Future prospective studies should include measurement of various inflammatory markers representing chronic inflammatory states. Third, potential covariates such as medications, hormonal status, and physical activity were not completely controlled due to the retrospective study design, although we excluded patients having chronic or severe medical illnesses.

In conclusion, patients with SSD and high BMI may exhibit increased somatic symptom severity, mediated by working memory dysfunction. Weight management may help improve symptoms in these patients. Future prospective studies, with larger sample sizes, are required.

\section{- Conflicts of Interest}

No potential conflict of interest relevant to this article was reported.

\section{Author Contributions}

Conceptualization: Sun Mi Kim, Hye Ri Kim. Data acquisition: Hee Jin Kim, Hye Ri Kim, Jae-Chan Jin. Formal analysis: Sun Mi Kim, Jae-Chan Jin. Supervision: Doug Hyun Han. Writing - original draft: Hee Jin Kim, Sun Mi Kim. Writing - review and editing: Doug Hyun Han. Approval of final manuscript: all authors.

\section{ORCID}

Hee Jin Kim https://orcid.org/0000-0002-8529-4786 Hye Ri Kim https://orcid.org/0000-0002-4147-4056 Jae-Chan Jin https://orcid.org/0000-0002-2419-5485 Doug Hyun Han https://orcid.org/0000-0001-5888-0686 Sun Mi Kim 


\section{REFERENCES}

1. American Psychiatric Association. Diagnostic and statistical manual of mental disorders: DSM-5. 5th ed. Arlington:American Psychiatric Publishing;2013.

2. Rief W, Mewes R, Martin A, Glaesmer H, Braehler E. Are psychological features useful in classifying patients with somatic symptoms? Psychosom Med 2010;72:648-655.

3. Barsky AJ, Orav EJ, Bates DW. Somatization increases medical utilization and costs independent of psychiatric and medical comorbidity. Arch Gen Psychiatry 2005;62:903-910.

4. Kurlansik SL, Maffei MS. Somatic symptom disorder. Am Fam Physician 2016;93:49-54.

5. Sadock BJ, Sadock VA. Kaplan \& Sadock's synopsis of psychiatry: behavioral sciences/clinical psychiatry. 10th ed. Philadelphia:Lippincott Williams \& Wilkins;2007.

6. Hall NM, Kuzminskyte R, Pedersen AD, Ørnbøl E, Fink P. The relationship between cognitive functions, somatization and behavioural coping in patients with multiple functional somatic symptoms. Nord J Psychiatry 2011;65:216-224.

7. Niemi PM, Portin R, Aalto S, Hakala M, Karlsson H. Cognitive functioning in severe somatization--a pilot study. Acta Psychiatr Scand 2002;106:461-463.

8. Martin A, Buech A, Schwenk C, Rief W. Memory bias for health-related information in somatoform disorders. J Psychosom Res 2007;63:663-671.

9. Rief W, Broadbent E. Explaining medically unexplained symptoms-models and mechanisms. Clin Psychol Rev 2007; 27:821-841.

10. Al-Adawi S, Al-Zakwani I, Obeid YA, Zaidan Z. Neurocognitive functioning in women presenting with undifferentiated somatoform disorders in Oman. Psychiatry Clin Neurosci 2010; 64:555-564.

11. Witthöft M, Gerlach AL, Bailer J. Selective attention, memory bias, and symptom perception in idiopathic environmental intolerance and somatoform disorders. J Abnorm Psychol 2006; 115:397-407.

12. Nikendei $C$, Waldherr S, Schiltenwolf $M$, Herzog W, Röhrig $\mathrm{M}$, Walther S, et al. Memory performance related to organic and psychosocial illness attributions in somatoform pain disorder patients. J Psychosom Res 2009;67:199-206.

13. Hakala M, Vahlberg T, Niemi PM, Karlsson H. Brain glucose metabolism and temperament in relation to severe somatization. Psychiatry Clin Neurosci 2006;60:669-675.

14. WHO Expert Consultation. Appropriate body-mass index for Asian populations and its implications for policy and intervention strategies. Lancet 2004;363:157-163.

15. Howard BV, Ruotolo G, Robbins DC. Obesity and dyslipidemia. Endocrinol Metab Clin North Am 2003;32:855-867.

16. DeFronzo RA, Ferrannini E. Insulin resistance. A multifaceted syndrome responsible for NIDDM, obesity, hypertension, dyslipidemia, and atherosclerotic cardiovascular disease. Diabetes Care 1991;14:173-194.
17. Rahmouni K, Correia ML, Haynes WG, Mark AL. Obesity-associated hypertension: new insights into mechanisms. Hypertension 2005;45:9-14.

18. Després JP, Lemieux I. Abdominal obesity and metabolic syndrome. Nature 2006;444:881-887.

19. Dixon JB. The effect of obesity on health outcomes. Mol Cell Endocrinol 2010;316:104-108.

20. Singh M. Mood, food, and obesity. Front Psychol 2014;5:925.

21. Reilly-Harrington NA, Feig EH, Huffman JC. Bipolar disorder and obesity: contributing factors, impact on clinical course, and the role of bariatric surgery. Curr Obes Rep 2018;7:294-300.

22. Mansur RB, Brietzke E, McIntyre RS, Cao B, Lee Y, Japiassú L, et al. BDNF and BMI effects on brain structures of bipolar offspring: results from the global mood and brain science initiative. Acta Psychiatr Scand 2017;136:607-614.

23. Xiang X, An R. Obesity and onset of depression among U.S. middle-aged and older adults. J Psychosom Res 2015;78:242248.

24. Martin-Rodriguez E, Guillen-Grima F, Aubá E, Martí A, Brugos-Larumbe A. Relationship between body mass index and depression in women: a 7-year prospective cohort study. The APNA study. Eur Psychiatry 2016;32:55-60.

25. Castanon N, Lasselin J, Capuron L. Neuropsychiatric comorbidity in obesity: role of inflammatory processes. Front Endocrinol (Lausanne) 2014;5:74.

26. Mansur RB, McIntyre RS, Cao B, Lee Y, Japiassú L, Chen K, et al. Obesity and frontal-striatal brain structures in offspring of individuals with bipolar disorder: results from the global mood and brain science initiative. Bipolar Disord 2018;20: 42-48.

27. Arvanitakis Z, Capuano AW, Bennett DA, Barnes LL. Body mass index and decline in cognitive function in older black and white persons. J Gerontol A Biol Sci Med Sci 2018;73: 198-203.

28. Arnsten AF, Rubia K. Neurobiological circuits regulating attention, cognitive control, motivation, and emotion: disruptions in neurodevelopmental psychiatric disorders. J Am Acad Child Adolesc Psychiatry 2012;51:356-367.

29. First MB, Williams JBW, Karg RS, Spitzer RL. User's guide for the structured clinical interview for DSM- $5^{\mathbb{R}}$ disorders: clinician version (SCID-5-CV). Arlington:American Psychiatric Publishing;2016.

30. Wechsler D. Wechsler Adult intelligence scale: fourth edition (WAIS - IV). 4th ed. San Antonio:Pearson;2008. p.22-498.

31. Hwang S, Kim J, Park K, Choi J, Hong S. Korean Wechsler adult intelligence scale-IV. 4th ed. Daegu:Korea Psychology Co.;2011.

32. Kwon S. Korean-symptom checklist II (KSCL95). Seoul:JungAng Aptitude Research Institute;2015. p.1-39.

33. Wium-Andersen MK, Ørsted DD, Nordestgaard BG. Elevated plasma fibrinogen, psychological distress, antidepressant use, and hospitalization with depression: two large populationbased studies. Psychoneuroendocrinology 2013;38:638-647. 
34. Beydoun MA, Beydoun HA, Dore GA, Canas JA, FanelliKuczmarski MT, Evans MK, et al. White blood cell inflammatory markers are associated with depressive symptoms in a longitudinal study of urban adults. Transl Psychiatry 2016;6:e895.

35. Baek JH, Lee $\mathrm{H}$, Myung W, Kim H, Choi YH, Kim DK, et al. The association between inflammatory markers and general psychological distress symptoms. Gen Hosp Psychiatry 2019; 56:9-12.

36. Euteneuer F, Schwarz MJ, Dannehl K, Hartung A, Westermann $\mathrm{S}$, Rief W. Increased soluble interleukin-2 receptor levels are related to somatic but not to cognitive-affective features in major depression. Brain Behav Immun 2012;26:1244-1248.

37. Dantzer R, O'Connor JC, Freund GG, Johnson RW, Kelley KW. From inflammation to sickness and depression: when the immune system subjugates the brain. Nat Rev Neurosci 2008; 9:46-56.

38. Capuron L, Miller AH. Immune system to brain signaling: neuropsychopharmacological implications. Pharmacol Ther 2011;130:226-238.

39. Kullmann S, Callaghan MF, Heni M, Weiskopf N, Scheffler K, Häring $\mathrm{HU}$, et al. Specific white matter tissue microstructure changes associated with obesity. Neuroimage 2016;125:36-44.

40. Alarcón G, Ray S, Nagel BJ. Lower working memory perform- ance in overweight and obese adolescents is mediated by white matter microstructure. J Int Neuropsychol Soc 2016;22: 281-292.

41. Kim SM, Hong JS, Min KJ, Han DH. Brain functional connectivity in patients with somatic symptom disorder. Psychosom Med 2019;81:313-318.

42. Luerding R, Weigand $T$, Bogdahn $U$, Schmidt-Wilcke $T$. Working memory performance is correlated with local brain morphology in the medial frontal and anterior cingulate cortex in fibromyalgia patients: structural correlates of pain-cognition interaction. Brain 2008;131(Pt 12):3222-3231.

43. Fougnie D. The relationship between attention and working memory. In: Johansen NB, editor. New research on short-term memory. New York:Nova Biomedical Books;2008. p. 1-45.

44. deCharms RC, Maeda F, Glover GH, Ludlow D, Pauly JM, Soneji $\mathrm{D}$, et al. Control over brain activation and pain learned by using real-time functional MRI. Proc Natl Acad Sci U S A 2005;102:18626-18631.

45. Seminowicz DA, Davis KD. A re-examination of pain-cognition interactions: implications for neuroimaging. Pain 2007; 130:8-13.

46. Kline RB. Principles and practice of structural equation modeling. 4th ed. New York:Guilford Press;2015. 